\title{
Hydrodynamic stress correlations in fluid films driven by stochastic surface forcing
}

\author{
Masoud Mohammadi-Arzanagh, ${ }^{1,2}$ Saeed Mahdisoltani, ${ }^{1,2}$ Rudolf Podgornik, ${ }^{3,4}$ and Ali Naji ${ }^{1}$, \\ ${ }^{1}$ School of Physics, Institute for Research in Fundamental Sciences (IPM), P.O. Box 19395-5531, Tehran, Iran \\ ${ }^{2}$ Department of Physics, Sharif University of Technology, P.O. Box 11155-9161, Tehran, Iran \\ ${ }^{3}$ School of Physical Sciences and Kavli Institute for Theoretical Sciences, \\ University of Chinese Academy of Sciences, Beijing 100049, China \\ ${ }^{4}$ CAS Key Laboratory of Soft Matter Physics, Institute of Physics, \\ Chinese Academy of Sciences, Beijing 100190, China
}

\begin{abstract}
We study hydrodynamic fluctuations in a compressible and viscous fluid film confined between two rigid, no-slip, parallel plates, where one of the plates is kept fixed, while the other one is driven in small-amplitude, translational, displacements around its reference position. This jiggling motion is assumed to be driven by a stochastic, external, surface forcing of zero mean and finite variance. Thus, while the transverse (shear) and longitudinal (compressional) hydrodynamic stresses produced in the film vanish on average on either of the plates, these stresses exhibit fluctuations that can be quantified through their equal-time, two-point, correlation functions. For transverse stresses, we show that the correlation functions of the stresses acting on the same plate (self-correlators) as well as the correlation function of the stresses acting on different plates (cross-correlators) exhibit universal, decaying, power-law behaviors as functions of the inter-plate separation. At small separations, the exponents are given by -1 , while at large separations, the exponents are found as -2 (self-correlator on the fixed plate), -4 (excess self-correlator on the mobile plate) and -3 (cross-correlator). For longitudinal stresses, we find much weaker power-law decays in the large separation regime, with exponents $-3 / 2$ (excess self-correlator on the mobile plate) and -1 (cross-correlator). The selfcorrelator on the fixed plate increases and levels off upon increasing the inter-plate separation, reflecting the non-decaying nature of the longitudinal forces acting on the fixed plate.
\end{abstract}

\section{INTRODUCTION}

Effective interactions between nano-/macromolecular bodies in aqueous solutions can broadly be decomposed into two equally important contributions: The static or equilibrium forces, and the dynamic forces, arising when the system is driven out of equilibrium, such as when the bodies are in relative motion [1, 2]. While the former originate in the disjoining pressure due to direct and/or solvent-mediated surface forces, operating primarily at the nanoscale, the latter depend on dynamic molecular processes in the solvent, and also on (slow) hydrodynamic stresses as the intervening solvent is drained/sheared from the liquid film separating the interacting surfaces. The dynamic forces can thus be relevant over a much wider range of nano-/microscale separations [1-4].

The extended Derjaguin-Landau-Verwey-Overbeek theory of colloidal stability identifies three types of static surface interactions [1, 2]: The electrostatic interactions depending on the specific nature of mobile and fixed molecular charges [5], the ubiquitous van-der-Waals (vdW) interactions, depending on the dielectric response of molecular materials [6], and solvent-mediated interactions stemming from the hydrophobic and/or hydration forces between solvent-exposed surfaces 7]. The dynamic forces are, on the other hand, much more difficult to classify unequivocally. Some of the equilibrium forces, as is the case with, for instance, the vdW interaction itself, can display an inherent dynamic component [8, 9]. Oth-

\footnotetext{
* Corresponding author: a.naji@ipm.ir
}

ers may exhibit no equilibrium counterpart as are the hydrodynamic interactions, having significant impact on dynamic properties (e.g., spatiotemporal correlations) of colloids in bulk [1,4] or strongly confined fluids [10, 11.

Recent advances in surface-force techniques, such as surface forces apparatus (SFA) and atomic force microscope (AFM) 1, 2, have enabled high-precision determination of both static and dynamic forces acting between contact surfaces across an intervening layer of simple or complex fluid (for recent reviews of relating techniques and applications, see Refs. 12 19]). Dynamic SFA usually incorporates two apposed, molecularly smooth, flattened or curved surfaces of relatively large radii of curvature, with one of the surfaces driven in controlled threedimensional (linear/oscillating) motion 12. This allows for measuring various (generally frequency-dependent) rheological properties of thin fluid films and gives direct access to shear/compressional forces exerted on the bounding surfaces by the intervening fluid over a wide range of surface separations and velocities/frequencies of the imposed surface motion [20 26]. Dynamic AFM has, on the other hand, emerged as an important tool for probing the local response of hard or soft material interfaces in liquid media [13 15. In colloidal-probe AFM [13, a relatively large, cantilever-mounted colloidal particle oscillates in proximity to an interface, with the power spectrum of the oscillations providing information on the hydrodynamic/viscoelastic properties of the surrounding liquid and the probe-surface interactions. The effects of oscillatory external forcing as well as thermal noise in dynamic AFM, involving colloidal probes or flat microlevers, have been analyzed on various levels of approx- 
imation [13, 27 31. In very recent works, Maali et al. have first analyzed the hydrodynamics of a vibrating microsphere [32] and then generalized the methodology to a thermally driven vibrating sphere yielding a thermalnoise AFM probe, where the sub-nanometer thermal motion of the sphere, coupled to a spring and dashpot mathematical model, reveals an (elasto)hydrodynamic coupling between the sphere and a vicinal, hard (mica) or soft (air-bubble), substrate in water 33.

While shear/drainage thin-film flows, caused by smallamplitude oscillatory or stochastic surface forcing [1216, 24 33, have been a common motif in the SFA/AFM contexts, other techniques for generating such flow patterns have been developed based on quartz crystal resonators (QCRs) to probe near-surface fluid properties [2, such as boundary slippage effects 17 19. QCRs are used (also in combination with the dynamic SFA [34]) as vibrating fluid substrates, driven at their resonance frequency to produce unsteady thin-film flows at high frequencies and shear rates (see the review in Ref. [18]).

In the aforementioned contexts, it is important to analyze first the well-defined limits and only then proceed to more advanced models to account for the various couplings and feedbacks 35. It is also important to realize that forced motion is in general incompatible with the assumptions of weak acceleration, requiring one to account for finite compressibility effects, which makes the underlying hydrodynamic problem more difficult to tackle 36].

Motivated by these advances, we formulate a general framework for surface-driven hydrodynamic interactions across a compressible and viscous fluid film, mechanically driven, in transverse/longitudinal directions, at one of its two rigid boundaries using an arbitrary external forcing. We focus on a fluid film with plane-parallel bounding surfaces, which is more yielding towards systematic calculations. Our primary interest is in the fluctuational behavior of shear/compressional hydrodynamic stresses generated across the film and on the boundaries, when the surface forcing is stochastic and of arbitrary (thermal or non-thermal) spectral density. The concrete example of temporally uncorrelated (white-noise) forcing will be analyzed numerically in detail. We show that the compressible, viscous hydrodynamic coupling between the mobile (forced) and the fixed bounding surfaces of the film leads to a complicated dependence of the same-plate and crossplate stress correlation functions on the inter-surface separation (film thickness). This includes decaying powerlaws with universal exponents and non-decaying stress variances on the fixed plate due to the acoustic resonances originating in the compressional modes.

This problem in some sense represents an inverse one with respect to the recently analyzed case of thermal fluctuating hydrodynamics [37] between two rigid plates, geared towards elucidating the possible role of the $h y$ drodynamic Casimir-like effects (in analogy with other examples of non-equilibrium fluctuation-induced forces involving fluctuating classical fields 38 48). The hydrodynamic Casimir-like phenomenon was in fact shown to exist only in its indirect "secondary" form [40: While the average stress on either of the bounding surfaces is zero, its fluctuations indicate long-range correlations, as a result of near-equilibrium thermal fluctuations in the confined fluid film. The forced fluctuations of one of the bounding plates of a confined fluid layer analyzed below are rather different from the hydrodynamic Casimireffect phenomenology, and are more closely related to the so-called Bjerknes interactions in driven acoustic resonators [49, 50]. Contrary to the Bjerknes interactions though, the forcing in our case is not due to a volumedistributed external acoustic field, but is rather exerted on one (or both) of the rigid bounding plates.

We introduce our framework in Section II and calculate the relevant hydrodynamic response/correlations for an arbitrary stochastic surface forcing in Section III. The numerical results are given for the special case of whitenoise forcing in Sections IV and $\mathrm{V}$, followed by the conclusions in Section VI.

\section{MODEL AND FORMALISM}

\section{A. Model geometry and physical description}

Let us consider a classical, compressible, viscous fluid film confined between two rigid, parallel plates of infinite extent in the $x-y$ coordinate plane at vertical locations $z=0$ and $z=h>0$; see Fig. 11. The upper plate is kept at rest, while an external surface force per unit area $\mathbf{f}=\mathbf{f}(t)$, drives the lower plate in arbitrary, translational, rigid-body movements around its reference plane at $z=0$. As a consequence the mobile plate exhibits a time-dependent surface velocity, $\mathbf{u}(t)$, which is to be determined consistently and concurrently with the fluid velocity, density and pressure fields within the film, i.e., $\mathbf{v}=\mathbf{v}(\mathbf{r} ; t), \rho=\rho(\mathbf{r} ; t)$, and $p=p(\mathbf{r} ; t)$, respectively.

In its general aspects, the present model is used to capture the elemental features of typical surface-driven flows in standard surface-force experiments [12 35], but it is also designed as a first-step model to facilitate an unequivocal elucidation of the basic physics of the problem using direct analytical calculations. It is nevertheless useful to detail the simplifying assumptions involved.

First, we note that while flattened/planar contact surfaces, as in our model, have been used in dynamic SFA/QCRs (see, e.g., Refs. [1, 2, 12, 20, 22, 34]), and also in dynamic AFM with wide flat microlevers [30, experiments often rely on cross-cylindrical or sphere-plane geometries. The radii of curvature in these applications are however very large (around a few $\mathrm{mm} / \mathrm{cm}$ in SFA and tens of $\mu \mathrm{m}$ in AFM) as compared to the film thickness (varied in the sub-nm to $\mu \mathrm{m}$ range); see, e.g., Refs. [1216, 20 28, 32, 33. For such weakly curved surfaces, the Derjaguin approximation [1, 2] can be used to predict the interaction forces between curved boundaries based merely on the results obtained in the plane-parallel geometry, or vice versa. 


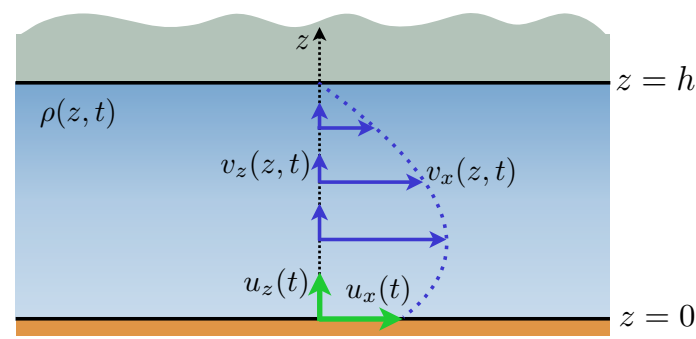

FIG. 1. Sideview of a plane-parallel film of a compressible, viscous fluid, driven at its lower boundary $(z=0)$ with an external forcing, giving the uniform surface velocity $\mathbf{u}(t)$.

Secondly, the amplitude of displacements (especially in $z$ direction) of the mobile plate is assumed here to be much smaller than the film thickness or, equivalently, $|\mathbf{u}(t)|$ is taken be sufficiently small [51]. This is in fact the typical situation also for the perpendicular (compressional), oscillatory or noise-driven, surface motions utilized in dynamic SFA/AFM experiments [12, 16, 24, 33]. It enables one to assume that the inter-plate separation is fixed on the leading order and also allows for a linearized treatment of the full Navier-Stokes equations [27+29, 31] by setting $\mathbf{v}=\mathbf{v}^{(1)}, p=p_{0}+p^{(1)}$ and $\rho=\rho_{0}+\rho^{(1)}$, where the superscript (1) denotes the first-order fluctuations around the rest values $\mathbf{v}=\mathbf{0}, p=p_{0}$ and $\rho=\rho_{0}$.

Thirdly, we neglect possible boundary slippage effects [17, 19, 24, 25, 30, 32, 33] by taking no-slip boundaries with $\mathbf{v}(x, y, z=0 ; t)=\mathbf{u}(t)$ and $\mathbf{v}(x, y, z=h ; t)=\mathbf{0}$.

Finally, we ignore local temperature variations and heat transfer processes in the film 52] (to be considered elsewhere [53]), the (nonlinear) viscous dissipation [23], and the relaxation effects that can formally be accounted for by taking frequency-dependent viscosities [37.

\section{B. Linearized fluid-film hydrodynamics}

The surface-driven flow problem described above is governed by the following set of equations to the first order in field fluctuations 37.

$$
\begin{gathered}
\eta \nabla^{2} \mathbf{v}^{(1)}+\left(\frac{\eta}{3}+\zeta\right) \nabla\left(\nabla \cdot \mathbf{v}^{(1)}\right)-\nabla p^{(1)}-\rho_{0} \partial_{t} \mathbf{v}^{(1)}=0, \\
\partial_{t} \rho^{(1)}+\rho_{0} \nabla \cdot \mathbf{v}^{(1)}=0, \quad p^{(1)}=c_{0}^{2} \rho^{(1)},
\end{gathered}
$$

where $c_{0}$ is the isothermal speed of sound [54]. These equations are supplemented by the no-slip boundary conditions $\mathbf{v}^{(1)}(x, y, z=0 ; t)=\mathbf{u}(t), \mathbf{v}^{(1)}(x, y, z=h ; t)=0$. The first-order hydrodynamic stress tensor is

$\sigma_{j k}^{(1)}=\eta\left[\nabla_{j} v_{k}^{(1)}+\nabla_{k} v_{j}^{(1)}\right]-\delta_{j k}\left[\left(\frac{2 \eta}{3}-\zeta\right) \nabla_{l} v_{l}^{(1)}+c_{0}^{2} \rho^{(1)}\right]$,

with $j, k, l=x, y, z$ denoting the Cartesian components.

We drop the superscript (1) for notational simplicity; thus, $\mathbf{v}(\mathbf{r} ; t), \rho(\mathbf{r} ; t)$, and $p(\mathbf{r} ; t)$ hereafter denote only the first-order field fluctuations around the given stationary values. Due to the one-dimensional nature of the flow, we drop the variables $x$ and $y$, and use the notation $\mathbf{v}=$ $\left(\mathbf{v}_{\|}, v_{z}\right), \mathbf{v}_{\|}=\left(v_{x}, v_{y}\right)$ to simplify Eqs. (1) and 22 as

$$
\begin{aligned}
& \rho_{0} \partial_{t} \mathbf{v}_{\|}=\eta \partial_{z}^{2} \mathbf{v}_{\|}, \\
& \rho_{0} \partial_{t} v_{z}=-c_{0}^{2} \partial_{z} \rho+\left(\frac{4 \eta}{3}+\zeta\right) \partial_{z}^{2} v_{z}, \\
& \partial_{t} \rho+\rho_{0} \partial_{z} v_{z}=0 .
\end{aligned}
$$

The different components of the velocity field are thus decoupled, as can be seen by combining Eqs. (5) and (6) to obtain the standard, attenuated wave equation

$$
\partial_{t}^{2} v_{z}=c_{0}^{2} \partial_{z}^{2} v_{z}+\nu_{\perp} \partial_{z}^{2} \partial_{t} v_{z}
$$

Due to the symmetry upon interchanging $v_{x}$ and $v_{y}$, we restrict our discussions of $\mathbf{v}_{\|}$only to its $v_{x}$ component. The relevant non-zero components of the stress tensor include only the transverse and longitudinal ones

$$
\begin{aligned}
\sigma_{x z}(z ; t) & =\rho_{0} \nu_{\|} \partial_{z} v_{x} \\
\sigma_{z z}(z ; t) & =\rho_{0} \nu_{\perp} \partial_{z} v_{z}-c_{0}^{2} \rho(z ; t),
\end{aligned}
$$

where $\nu_{\|}=\eta / \rho_{0}$ and $\nu_{\perp}=(4 \eta / 3+\zeta) / \rho_{0}$ are the corresponding transverse (shear) and longitudinal (compressional) kinematic viscosities, respectively.

Since our formulation is linear, the solutions reported below can linearly be superposed to study the case with both lower and upper plates undergoing small-amplitude displacements. This is a straightforward generalization, which we shall not discuss any further.

\section{Equation of motion for the mobile surface}

Since the lower (mobile) plate is driven by the external force per unit area, $\mathbf{f}=\mathbf{f}(t)$, causing it to move with the velocity $\mathbf{u}=\mathbf{u}(t)$, we can write Newton's second law for its motion in the frequency (Fourier) domain as

$$
-i m \omega \widetilde{u}_{j}(\omega)=\widetilde{f}_{j}(\omega)-\widetilde{\sigma}_{j z}(z=0 ; \omega) n_{z}, \quad j=x, z,
$$

where $m$ is the plate mass per unit area, and $\widetilde{f}_{j}$ and $\widetilde{u}_{j}$ are the frequency-domain components of $\mathbf{f}$ and $\mathbf{u}$, respectively. Here, $n_{z}=-1$ is the $z$ component of the unit vector along the inward normal to the mobile plate, making $-\widetilde{\sigma}_{j z}(z=0 ; \omega) n_{z}$ the force component per unit area acting on the plate due to hydrodynamic stresses 37.

We solve Eqs. (5) and (6) with the required boundary conditions to find the solutions for the fluid velocity/density fields in terms of the surface velocity $\mathbf{u}(t)$, which can itself be determined as a function of $\mathbf{f}(t)$ by inserting those solutions into the stress term in Eq. 10. This gives the the desired final forms of the fluid velocity/density fields as functions of the external forcing $\mathbf{f}(t)$.

Our formulation can be implemented with any surface forcing model of either deterministic or stochastic origins. Stochastic forcing is particularly relevant 
to the thermal-noise AFM probe [33, resulting, e.g., from Brownian fluctuations in the setup or ambient fluid. We shall adopt a Gaussian-distributed stochastic forcing with mean $\left\langle\widetilde{f}_{j}(\omega)\right\rangle=0$ and two-point correlation function $\left\langle\widetilde{f}_{j}(\omega) \widetilde{f}_{k}\left(\omega^{\prime}\right)\right\rangle=4 \pi \widetilde{\mathcal{G}}_{j}(\omega) \delta_{j k} \delta\left(\omega+\omega^{\prime}\right)$, where $\widetilde{\mathcal{G}}_{j}(\omega)$ are the real-valued and positive forcing spectral densities. In our numerical analysis later, we shall adopt the white-noise Ansatz with $\widetilde{\mathcal{G}}_{j}(\omega)=\mathcal{G}_{j}$ (of dimension $[\text { pressure }]^{2} \cdot[$ time]) taken as constants.

Note that the zero mean taken for the surface forcing implies that the hydrodynamic stresses acting on the plates due to the fluctuations in the film will be zero on average, $\left\langle\sigma_{x z}(z ; t)\right\rangle=\left\langle\sigma_{z z}(z ; t)\right\rangle=0$, where the brackets $\langle\cdots\rangle$ denote ensemble averaging over various realizations of the external forcing. Yet, the variance and correlation functions (or correlators) of instantaneous stresses can be finite, characterizing the measurable fluctuation-induced forces mediated by hydrodynamic correlations between the plates on the leading order. Although, these forces resemble the secondary hydrodynamic Casimir-like forces arising from near-equilibrium thermal fluctuations [40], it is important to note that the stochastic forcing here can generally be non-thermal, in which case it can produce far-from-equilibrium stress fluctuations and correlations in the fluid film. To evaluate these correlations, we first give the solutions to the velocity and density fields in terms of the stress response functions of the fluid film.

\section{RESPONSE TO SURFACE FORCING}

\section{A. Velocity and density fields}

The velocity and density field fluctuations can be obtained by transforming Eqs. (4) and (7) to the frequency domain. The governing equations for transverse and longitudinal modes read, respectively, as

$$
\begin{aligned}
& \partial_{z}^{2} \widetilde{v}_{x}(z ; \omega)=-\alpha^{2}(\omega) \widetilde{v}_{x}(z ; \omega) \\
& \partial_{z}^{2} \widetilde{v}_{z}(z ; \omega)=-\kappa^{2}(\omega) \widetilde{v}_{z}(z ; \omega)
\end{aligned}
$$

where $\alpha^{2}(\omega)=i \omega / \nu_{\|}$and $\kappa^{2}(\omega)=\omega^{2} /\left(c_{0}^{2}-i \omega \nu_{\perp}\right)$. Here, $\alpha$ and $\kappa$ give the frequency-dependent (screening) length-scales associated with the shear and compressional modes, respectively. There will be two equivalent sets of solutions for $\alpha$ and $\kappa$, fulfilling the relations $\alpha^{*}(\omega)= \pm \alpha(-\omega), \kappa^{*}(\omega)= \pm \kappa(-\omega)$. One can conveniently choose the solutions satisfying these relations with plus signs, giving real (R) and imaginary (I) parts

$$
\begin{aligned}
& \alpha_{\mathrm{R}}(\omega)=\alpha_{\mathrm{I}}(\omega) \operatorname{sgn}(\omega)= \pm \sqrt{\frac{|\omega|}{2 \nu_{\|}}}, \\
& \kappa_{\mathrm{R}}(\omega)= \pm \frac{|\omega|}{\sqrt{2}} \sqrt{\frac{\sqrt{c_{0}^{4}+\omega^{2} \nu_{\perp}^{2}}+c_{0}^{2}}{c_{0}^{4}+\omega^{2} \nu_{\perp}^{2}}} \\
& \kappa_{\mathrm{I}}(\omega)= \pm \frac{\omega}{\sqrt{2}} \sqrt{\frac{\sqrt{c_{0}^{4}+\omega^{2} \nu_{\perp}^{2}}-c_{0}^{2}}{c_{0}^{4}+\omega^{2} \nu_{\perp}^{2}}}
\end{aligned}
$$

where $\operatorname{sgn}(\cdot)$ is the sign function. Now, solving Eqs. 111 and $(12)$ with the required boundary conditions gives

$$
\begin{aligned}
& v_{x}(z ; t)=\int \frac{\mathrm{d} \omega}{2 \pi} e^{-i \omega t} \frac{\sin [\alpha(\omega)(h-z)]}{\sin [\alpha(\omega) h]} \widetilde{u}_{x}(\omega), \\
& v_{z}(z ; t)=\int \frac{\mathrm{d} \omega}{2 \pi} e^{-i \omega t} \frac{\sin [\kappa(\omega)(h-z)]}{\sin [\kappa(\omega) h]} \widetilde{u}_{z}(\omega) .
\end{aligned}
$$

The density field fluctuations can be obtained using Eqs. (6) and Eq. (17), yielding

$$
\rho(z ; t)=\rho_{0} \int \frac{\mathrm{d} \omega}{2 \pi} e^{-i \omega t}\left[\frac{i \kappa(\omega)}{\omega}\right] \frac{\cos [\kappa(\omega)(h-z)]}{\sin [\kappa(\omega) h]} \widetilde{u}_{z}(\omega) .
$$

It should be noted that the homogeneous parts of the solutions (in the time domain) are discarded as they depend on initial conditions, being irrelevant in the longtime stationary state to be studied here.

\section{B. Response and correlation functions}

Plugging Eqs. (16)-18 into Eqs. (8) and (9), we can write the two components of the surface stress tensor, $\tilde{\sigma}_{j z}(z=0 ; \omega)$, in terms of the surface velocity components $\widetilde{u}_{j}(\omega)$ for $j=x, z$. Inserting the results into Eq. (10), we find $\widetilde{u}_{j}(\omega)$ in terms of the surface forcing components, $\tilde{f}_{j}(\omega)$ and, then, using Eqs. 16 and (17), find the velocity field components $v_{j}(z ; t)$ in terms of $\widetilde{f}_{j}(\omega)$ as

$$
v_{j}(z ; t)=\int \frac{\mathrm{d} \omega}{2 \pi} e^{-i \omega t} \widetilde{R}_{j}(z ; \omega) \widetilde{f}_{j}(\omega),
$$

where the velocity response functions are given by

$$
\begin{aligned}
\widetilde{R}_{x}(z ; \omega) & =\frac{\sin [\alpha(\omega)(h-z)]}{\rho_{0} \nu_{\|} \alpha(\omega)\left(\cos [\alpha(\omega) h]-\frac{m}{\rho_{0}} \alpha(\omega) \sin [\alpha(\omega) h]\right)}, \\
\widetilde{R}_{z}(z ; \omega) & =\frac{\sin [\kappa(\omega)(h-z)]}{\rho_{0} \nu_{\perp}\left(1+i \frac{c_{0}^{2}}{\nu_{\perp} \omega}\right) \kappa(\omega)\left(\cos [\kappa(\omega) h]-\frac{m}{\rho_{0}} \kappa(\omega) \sin [\kappa(\omega) h]\right)}
\end{aligned}
$$


These play the role of the Oseen tensor components in the considered geometry. We can now express the stress tensor components in terms of the external forcing as

$$
\sigma_{j z}(z ; t)=\int \frac{\mathrm{d} \omega}{2 \pi} e^{-i \omega t} \widetilde{\chi}_{j z}(z ; \omega) \tilde{f}_{j}(\omega),
$$

where the stress response functions (analogous to the pressure vector for the Oseen problem [4]) are given by

$$
\begin{aligned}
& \widetilde{\chi}_{x z}(z ; \omega)=\rho_{0} \nu_{\|} \partial_{z} \widetilde{R}_{x}(z ; \omega), \\
& \widetilde{\chi}_{z z}(z ; \omega)=\rho_{0} \nu_{\perp}\left(1+i \frac{c_{0}^{2}}{\nu_{\perp} \omega}\right) \partial_{z} \widetilde{R}_{z}(z ; \omega) .
\end{aligned}
$$

Equations (22)-24 can be used to evaluate the desired two-point correlators of the stresses across the fluid film $\left(0 \leq z, z^{\prime} \leq h\right)$ defined as

$$
\mathcal{C}_{j z}\left(z, z^{\prime} ; t-t^{\prime}\right)=\left\langle\sigma_{j z}(z ; t) \sigma_{j z}\left(z^{\prime} ; t^{\prime}\right)\right\rangle
$$

for $j=x, z$, where the time homogeneity of the correlators in the stationary state is also explicitly indicated. The average in Eq. 25 can be evaluated (Section II C), yielding the transverse/longitudinal stress correlators in the frequency domain in terms of the stress response functions 23 and (24) as

$$
\widetilde{\mathcal{C}}_{j z}\left(z, z^{\prime} ; \omega\right)=2 \widetilde{\mathcal{G}}_{j}(\omega) \widetilde{\chi}_{j z}(z ; \omega) \widetilde{\chi}_{j z}\left(z^{\prime} ;-\omega\right) .
$$

Other relevant quantities include the two-point correlators of density and pressure fluctuations expressed as

$$
\begin{aligned}
& \mathcal{C}_{\rho \rho}\left(z, z^{\prime} ; t-t^{\prime}\right)=\left\langle\rho(z ; t) \rho\left(z^{\prime} ; t^{\prime}\right)\right\rangle, \\
& \mathcal{C}_{p p}\left(z, z^{\prime} ; t-t^{\prime}\right)=\left\langle p(z ; t) p\left(z^{\prime} ; t^{\prime}\right)\right\rangle .
\end{aligned}
$$

These quantities are related through $\mathcal{C}_{p p}=\mathcal{C}_{\rho \rho} / c_{0}^{4}$ (see Eq. (2)). In the present context, the frequency-domain forms (or, the spectral densities) of density correlator, $\widetilde{\mathcal{C}}_{\rho \rho}\left(z, z^{\prime} ; \omega\right)$, and the corresponding pressure correlator, $\widetilde{\mathcal{C}}_{p p}\left(z, z^{\prime} ; \omega\right)$, are found to be related directly to that of the longitudinal stress correlator as

$$
\widetilde{\mathcal{C}}_{\rho \rho}\left(z, z^{\prime} ; \omega\right)=c_{0}^{-4} \widetilde{\mathcal{C}}_{p p}\left(z, z^{\prime} ; \omega\right)=\frac{\widetilde{\mathcal{C}}_{z z}\left(z, z^{\prime} ; \omega\right)}{c_{0}^{4}+\nu_{\perp}^{2} \omega^{2}} .
$$

The two-point density correlator is of special interest in the context of thermal (near-equilibrium) hydrodynamic fluctuations in bulk fluids, in which case one uses its frequency/wavevector representation to obtain the spectral density of density fluctuations [55, 56. This latter quantity can be measured through inelastic (polarized) light scattering methods, enabling experimental determination of hydrodynamic-fluctuation spectra as well as various thermodynamic quantities and transport coefficients of bulk fluids. Our formulation directly relates the spectral density of hydrodynamic stress fluctuations on the film boundaries to the spectral density of fluid density fluctuations within the film. As such, it suggests an alternative method to probe the density fluctuations through the measurements of surface forces in confined fluids, where standard light scattering methods may be less suitable.

\section{Dimensionless representation}

In our later treatment of shear (\|) and compressional $(\perp)$ modes in dimensionless units, we shall make use of the rescaled variables

$$
z_{\|}=\frac{z}{\nu_{\|, \perp} / c_{0}}, \quad \tau_{\|}=\frac{t}{\nu_{\|, \perp} / c_{0}^{2}} \quad \text { and } \quad \omega_{\|}=\frac{\omega}{c_{0}^{2} / \nu_{\|, \perp}},
$$

as well as the rescaled inter-plate separation (rescaled film thickness) and the dimensionless mass per unit area of the mobile plate, respectively, as

$$
h_{\|}=\frac{h}{\nu_{\|, \perp} / c_{0}} \quad \text { and } \quad \gamma_{\|}=\frac{m}{\rho_{0} \nu_{\|, \perp} / c_{0}} .
$$

Using the definitions in Eqs. 13)-15, the parameter $\alpha(\omega)$ can be rescaled as $\nu_{\|} \alpha(\omega) \bar{c}_{0} \rightarrow \xi\left(\omega_{\|}\right)$, where

$$
\xi\left(\omega_{\|}\right)= \pm \sqrt{\frac{\left|\omega_{\|}\right|}{2}}\left(1+i \operatorname{sgn}\left(\omega_{\|}\right)\right)
$$

while $\kappa(\omega)$ can be rescaled as $\nu_{\perp} \kappa(\omega) / c_{0} \rightarrow \ell\left(\omega_{\perp}\right)=$ $\ell_{\mathrm{R}}\left(\omega_{\perp}\right)+i \ell_{\mathrm{I}}\left(\omega_{\perp}\right)$, with real and imaginary parts

$$
\begin{aligned}
& \ell_{\mathrm{R}}\left(\omega_{\perp}\right)= \pm \frac{\left|\omega_{\perp}\right|}{\sqrt{2}} \sqrt{\frac{\sqrt{1+\omega_{\perp}^{2}}+1}{1+\omega_{\perp}^{2}}}, \\
& \ell_{\mathrm{I}}\left(\omega_{\perp}\right)= \pm \frac{\omega_{\perp}}{\sqrt{2}} \sqrt{\frac{\sqrt{1+\omega_{\perp}^{2}}-1}{1+\omega_{\perp}^{2}}} .
\end{aligned}
$$

The spectral densities of external surface forcing are rescaled as $\widetilde{\mathcal{G}}_{x}(\omega) / \mathcal{G}_{x} \rightarrow \widetilde{\mathcal{G}}_{\|}\left(\omega_{\|}\right)$and $\widetilde{\mathcal{G}}_{z}(\omega) / \mathcal{G}_{z} \rightarrow \widetilde{\mathcal{G}}_{\perp}\left(\omega_{\perp}\right)$.

The transverse and longitudinal stress response functions 23 and 24 are dimensionless and can be re-expressed in rescaled coordinates immediately as $\tilde{\chi}_{x z}(z ; \omega) \rightarrow \widetilde{\chi}_{\|}\left(z_{\|} ; \omega_{\|}\right)$and $\tilde{\chi}_{z z}(z ; \omega) \rightarrow \widetilde{\chi}_{\perp}\left(z_{\perp} ; \omega_{\perp}\right)$ with explicit forms given by

$$
\begin{aligned}
& \tilde{\chi}_{\|}\left(z_{\|} ; \omega_{\|}\right)=-\frac{\cos \left[\xi\left(\omega_{\|}\right)\left(h_{\|}-z_{\|}\right)\right]}{\cos \left[\xi\left(\omega_{\|}\right) h_{\|}\right]-\gamma_{\|} \xi\left(\omega_{\|}\right) \sin \left[\xi\left(\omega_{\|}\right) h_{\|}\right]}, \\
& \tilde{\chi}_{\perp}\left(z_{\perp} ; \omega_{\perp}\right)=-\frac{\cos \left[\ell\left(\omega_{\perp}\right)\left(h_{\perp}-z_{\perp}\right)\right]}{\cos \left[\ell\left(\omega_{\perp}\right) h_{\perp}\right]-\gamma_{\perp} \ell\left(\omega_{\perp}\right) \sin \left[\ell\left(\omega_{\perp}\right) h_{\perp}\right]} .
\end{aligned}
$$

The transverse and longitudinal stress correlators in the time domain, Eq. (25), are rescaled by the characteristic stress variances $2 \mathcal{G}_{x} c_{0}^{2} / \nu_{\|}$and $2 \mathcal{G}_{z} c_{0}^{2} / \nu_{\perp}$ as

$$
\begin{aligned}
& \frac{\mathcal{C}_{x z}\left(z, z^{\prime} ; t-t^{\prime}\right)}{2 \mathcal{G}_{x} c_{0}^{2} / \nu_{\|}} \rightarrow \mathcal{C}_{\|}\left(z_{\|}, z_{\|}^{\prime} ; \Delta \tau_{\|}\right), \\
& \frac{\mathcal{C}_{z z}\left(z, z^{\prime} ; t-t^{\prime}\right)}{2 \mathcal{G}_{z} c_{0}^{2} / \nu_{\perp}} \rightarrow \mathcal{C}_{\perp}\left(z_{\perp}, z_{\perp}^{\prime} ; \Delta \tau_{\perp}\right),
\end{aligned}
$$

where we have defined $\Delta \tau_{\perp}=\tau_{\perp}-\tau_{\perp}^{\prime}$ and $\Delta \tau_{\|}=\tau_{\|}-\tau_{\|}^{\prime}$. These time-dependent (real-valued) stress correlators are 
to be calculated from the Fourier-transform relations

$$
\begin{aligned}
& \mathcal{C}_{\|}\left(z_{\|}, z_{\|}^{\prime} ; \Delta \tau_{\|}\right)=\int \frac{\mathrm{d} \omega_{\|}}{2 \pi} e^{-i \omega_{\|} \Delta \tau_{\|}} \widetilde{\mathcal{C}}_{\|}\left(z_{\|}, z_{\|}^{\prime} ; \omega_{\|}\right), \\
& \mathcal{C}_{\perp}\left(z_{\perp}, z_{\perp}^{\prime} ; \Delta \tau_{\perp}\right)=\int \frac{\mathrm{d} \omega_{\perp}}{2 \pi} e^{-i \omega_{\perp} \Delta \tau_{\perp} \widetilde{\mathcal{C}}_{\perp}}\left(z_{\perp}, z_{\perp}^{\prime} ; \omega_{\perp}\right),
\end{aligned}
$$

and also by making use of the frequency-domain expressions for $\widetilde{\mathcal{C}}_{\|}\left(z_{\|}, z_{\|}^{\prime} ; \omega_{\|}\right)$and $\widetilde{\mathcal{C}}_{\perp}\left(z_{\perp}, z_{\perp}^{\prime} ; \omega_{\perp}\right)$, themselves following from the dimensionless form of Eq. (26) as

$$
\begin{aligned}
& \widetilde{\mathcal{C}}_{\|}\left(z_{\|}, z_{\|}^{\prime} ; \omega_{\|}\right)=\widetilde{\mathcal{G}}_{\|}\left(\omega_{\|}\right) \widetilde{\chi}_{\|}\left(z_{\|} ; \omega_{\|}\right) \widetilde{\chi}_{\|}\left(z_{\|}^{\prime} ;-\omega_{\|}\right), \\
& \widetilde{\mathcal{C}}_{\perp}\left(z_{\perp}, z_{\perp}^{\prime} ; \omega_{\perp}\right)=\widetilde{\mathcal{G}}_{\perp}\left(\omega_{\perp}\right) \widetilde{\chi}_{\perp}\left(z_{\perp} ; \omega_{\perp}\right) \widetilde{\chi}_{\perp}\left(z_{\perp}^{\prime} ;-\omega_{\perp}\right) .
\end{aligned}
$$

Since $\xi\left(-\omega_{\|}\right)=\xi^{*}\left(\omega_{\|}\right)$and $\ell\left(-\omega_{\perp}\right)=\ell^{*}\left(\omega_{\perp}\right)$ (Section III A and, hence, $\tilde{\chi}_{\|}\left(z_{\|} ;-\omega_{\|}\right)=\tilde{\chi}_{\|}^{*}\left(z_{\|} ; \omega_{\|}\right)$and $\tilde{\chi}_{\perp}\left(z_{\|} ;-\omega_{\|}\right)=\tilde{\chi}_{\perp}^{*}\left(z_{\|} ; \omega_{\|}\right)$, the stress correlators are found to be symmetric w.r.t. interchanging their spatial (first and second) arguments and concurrently reversing the sign of their frequency/time (third) argument (or, by taking their complex conjugates); i.e., $\widetilde{\mathcal{C}}_{\|}\left(z_{\|}, z_{\|}^{\prime} ; \omega_{\|}\right)=$ $\widetilde{\mathcal{C}_{\|}}\left(z_{\|}^{\prime}, z_{\|} ;-\omega_{\|}\right)=\widetilde{\mathcal{C}}_{\|}^{*}\left(z_{\|}^{\prime}, z_{\|} ; \omega_{\|}\right)$, and $\widetilde{\mathcal{C}}_{\perp}\left(z_{\perp}, z_{\perp}^{\prime} ; \omega_{\perp}\right)=$ $\widetilde{\mathcal{C}}_{\perp}\left(z_{\perp}^{\prime}, z_{\perp} ;-\omega_{\perp}\right)=\widetilde{\mathcal{C}}_{\perp}^{*}\left(z_{\perp}^{\prime}, z_{\perp} ; \omega_{\perp}\right)$. Formally similar symmetry relations hold in the time domain for $\mathcal{C}_{\|}\left(z_{\|}, z_{\|}^{\prime} ; \Delta \tau_{\|}\right)$ and $\mathcal{C}_{\perp}\left(z_{\perp}, z_{\perp}^{\prime} ; \Delta \tau_{\perp}\right)$, and also for the density and pressure correlators as implied by Eq. (29). It should also be noted that the same-point correlators, $\widetilde{\mathcal{C}}_{\|}\left(z_{\|}, z_{\|} ; \omega_{\|}\right)$ and $\widetilde{\mathcal{C}}_{\perp}\left(z_{\perp}, z_{\perp} ; \omega_{\perp}\right)$, are nothing but the local variances of stress fluctuations at a given frequency. They are evidently real-valued and positive and, as such, ensure the stability of our linear-fluctuation analysis.

For the sake of concreteness in our numerical analysis below, we set the forcing spectral densities equal to one, $\widetilde{\mathcal{G}_{\|}}\left(\omega_{\|}\right)=\widetilde{\mathcal{G}}_{\perp}\left(\omega_{\perp}\right)=1$, equivalent to adopting the whitenoise Ansatz for the surface forcing as noted before.

\section{TRANSVERSE STRESS CORRELATORS}

We start our analysis by focusing first on the transverse hydrodynamic stresses produced by the white noise external surface forcing. The two-point correlator in the frequency domain, $\widetilde{\mathcal{C}_{\|}}\left(z_{\|}, z_{\|}^{\prime} ; \omega_{\|}\right)$, can be shown to be only a function of the redefined dimensionless frequency and coordinate variables $\omega_{\|} h_{\|}^{2}$ and $z_{\|} / h_{\|}$and the rescaled parameter $h_{\|} / \gamma_{\|}$. By setting $z_{\|}, z_{\|}^{\prime}=\left\{0, h_{\|}\right\}$, we obtain the same-plate and cross-plate correlators, which we shall refer to as self- and cross-correlators, respectively, as

$$
\begin{aligned}
& \widetilde{\mathcal{C}}_{\|}\left(0,0 ; \omega_{\|}\right)=\left|\widetilde{\chi}_{\|}\left(0 ; \omega_{\|}\right)\right|^{2}, \widetilde{\mathcal{C}}_{\|}\left(h_{\|}, h_{\|} ; \omega_{\|}\right)=\left|\widetilde{\chi}_{\|}\left(h_{\|} ; \omega_{\|}\right)\right|^{2}, \\
& \widetilde{\mathcal{C}}_{\|}\left(0, h_{\|} ; \omega_{\|}\right)=\widetilde{\mathcal{C}}_{\|}^{*}\left(h_{\|}, 0 ; \omega_{\|}\right)=\widetilde{\chi}_{\|}\left(0 ; \omega_{\|}\right) \widetilde{\chi}_{\|}^{*}\left(h_{\|} ; \omega_{\|}\right) .
\end{aligned}
$$

In Fig. 22 we show the self-correlators, $\widetilde{\mathcal{C}_{\|}}\left(0,0 ; \omega_{\|}\right)$and $\widetilde{\mathcal{C}_{\|}}\left(h_{\|}, h_{\|} ; \omega_{\|}\right)$, and the real part of the cross-correlator,

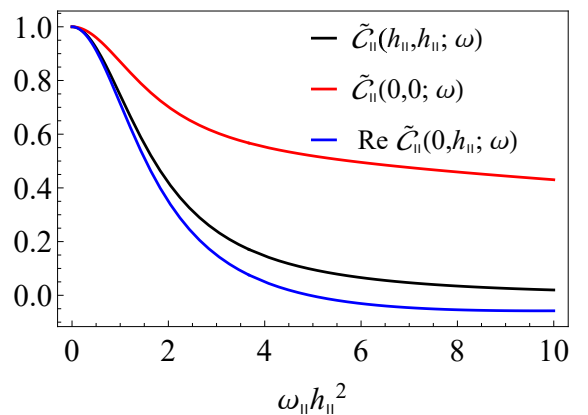

FIG. 2. The self- and cross-correlators of the transverse stresses acting on the plates as functions of the redefined dimensionless frequency, $\omega_{\|} h_{\|}^{2}$, for fixed $h_{\|} / \gamma_{\|}=5$.

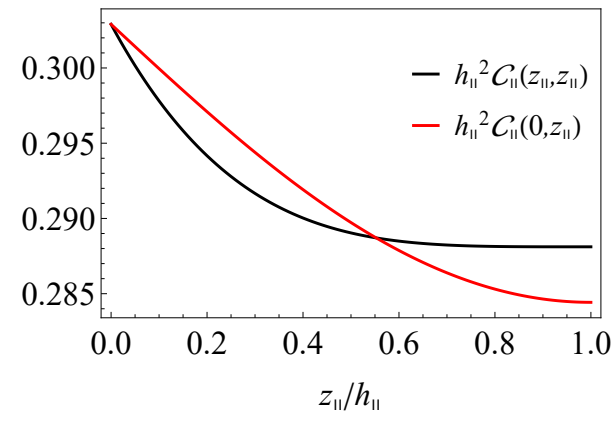

FIG. 3. Profiles of the rescaled, equal-time, transverse stress correlators $h_{\|}^{2} \mathcal{C}_{\|}\left(z_{\|}, z_{\|}\right)$and $h_{\|}^{2} \mathcal{C}_{\|}\left(0, z_{\|}\right)$across the fluid film $\left(0 \leq z_{\|} \leq h_{\|}\right)$for fixed $h_{\|} / \gamma_{\|}=5$.

$\operatorname{Re} \widetilde{\mathcal{C}}_{\|}\left(0, h_{\|} ; \omega_{\|}\right)$(being the only part that contributes to the cross-correlator in the time domain) as functions of $\omega_{\|} h_{\|}^{2}$ for $h_{\|} / \gamma_{\|}=5$. As seen, the three quantities exhibit rapid monotonic decrease with the frequency from their maximum value of one at zero frequency fulfilling the inequalities $\operatorname{Re} \widetilde{\mathcal{C}_{\|}}\left(0, h_{\|} ; \omega_{\|}\right) \leq \widetilde{\mathcal{C}}_{\|}\left(h_{\|}, h_{\|} ; \omega_{\|}\right) \leq \widetilde{\mathcal{C}_{\|}}\left(0,0 ; \omega_{\|}\right)$ across the frequency domain.

These behaviors can be understood by noting that the characteristic frequencies of the transverse modes occur at the poles of the transverse response function $\widetilde{\chi}_{\|}$over the complex frequency ( $\varsigma$ ) plane. These poles are found to fall onto the lower-half imaginary axis, reflecting the diffusive nature of the shear modes in the hydrodynamic regime (Appendix A). The singular part of the transverse response functions in the proximity of a given pole $\varsigma_{\|}^{(n)}$ (see Eq. A3 ) behaves as $\widetilde{\chi}_{\|} \sim 1 /\left(\omega_{\|}^{2}+\left[\operatorname{Im} \varsigma-\operatorname{Im} \varsigma_{\|}^{(n)}\right]^{2}\right)$. Thus, along the real-valued frequency axis $\omega_{\|}=\operatorname{Re} \varsigma, \widetilde{\chi}_{\|}$ takes a Lorentzian form peaked around $\omega_{\|}=0$ as all of the poles are purely imaginary, explaining the monotonically decreasing behaviors in Fig. 2 . 

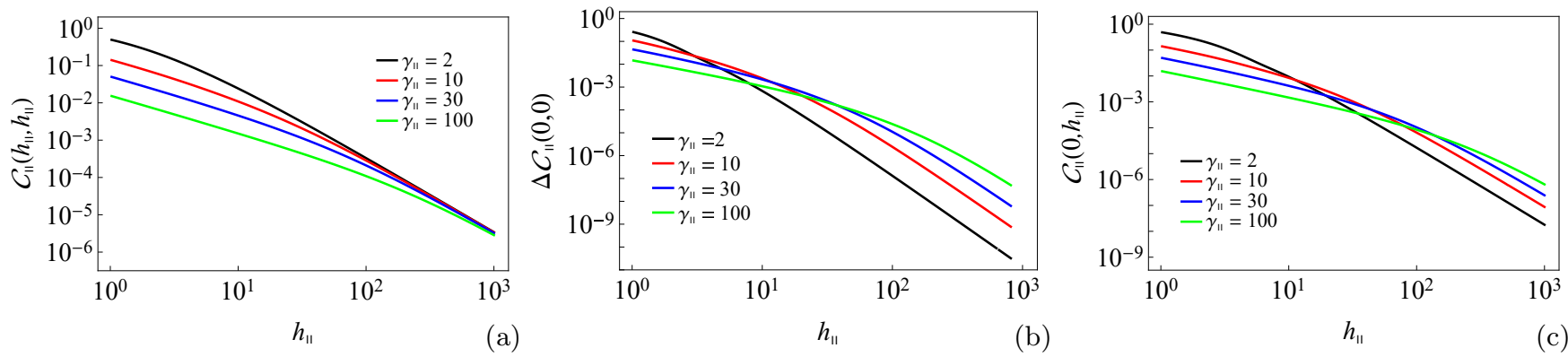

FIG. 4. Log-log plots of equal-time transverse stress (self- and cross-) correlators $\mathcal{C}_{\|}\left(h_{\|}, h_{\|}\right), \Delta \mathcal{C}_{\|}(0,0)$ and $\mathcal{C}_{\|}\left(0, h_{\|}\right)$, in panels (a), (b) and (c), respectively, as functions of the dimensionless inter-plate separation, $h_{\|}$, for $\gamma_{\|}=2,10,30$ and 100 .

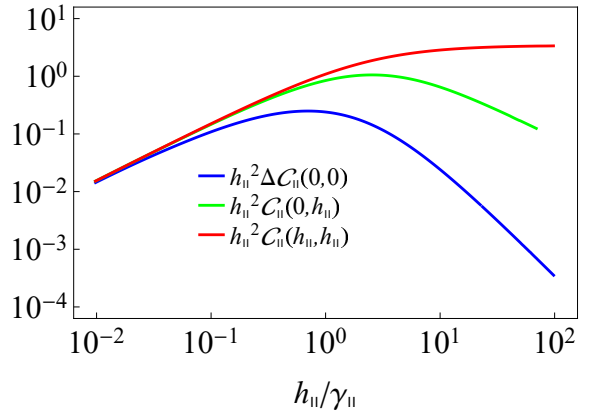

FIG. 5. The rescaled universal forms of the three equal-time transverse stress correlators, as indicated on the graph (see also Figs. 4 a-c), as functions of $h_{\|} / \gamma_{\|}$, demonstrating the crossover between the two power-law regimes found at small and large values of $h_{\|} / \gamma_{\|}$in each case.

\section{A. Equal-time transverse stress correlators}

The equal-time transverse correlators are obtained by setting $\Delta \tau_{\|}=0$ in Eq. 39, in which case we drop the time (third) argument by redefining our notation as $\mathcal{C}_{\|}\left(z_{\|}, z_{\|}^{\prime}\right) \equiv \mathcal{C}_{\|}\left(z_{\|}, z_{\|}^{\prime} ; \Delta \tau_{\|}=0\right)$. Hence,

$$
\mathcal{C}_{\|}\left(z_{\|}, z_{\|}^{\prime}\right)=\int \frac{\mathrm{d} \omega_{\|}}{2 \pi} \widetilde{\chi}_{\|}\left(z_{\|} ; \omega_{\|}\right) \tilde{\chi}_{\|}^{*}\left(z_{\|}^{\prime} ; \omega_{\|}\right) .
$$

When rescaled with the inter-plate separation as $h_{\|}^{2} \mathcal{C}_{\|}\left(z_{\|}, z_{\|}\right)$and $h_{\|}^{2} \mathcal{C}_{\|}\left(0, z_{\|}\right)$, these correlators will be functions of $z_{\|} / h_{\|}$and $h_{\|} / \gamma_{\|}$only. As seen in Fig. 3. both of these correlators vary within a relatively smant range of values across the film. Although this may naively appear as indicating an approximate power-law behavior of $\mathcal{C}_{\|} \sim h_{\|}^{-2}$ at any point across the film, we find a more diverse set of power-law behaviors for the self- and crosscorrelators of the stresses acting on the plates.

It should be noted that the frequency integrals discussed above converge sufficiently rapidly and, therefore, remain finite. For numerical expediency, and as a check on the self-consistency of the continuum approach used here (see Appendix B for details), it is convenient to introduce a high frequency cutoff $\omega_{\|}^{\infty}$, which (as discussed in the appendix) can conveniently be taken as $\omega_{\|}^{\infty}=1$. In general, the numerical outcomes for $\mathcal{C}_{\|}\left(h_{\|}, h_{\|}\right)$and $\mathcal{C}_{\|}\left(0, h_{\|}\right)$remain unaffected by the precise choice of the frequency cutoff, when the latter is sufficiently large. The self-correlator on the mobile (lower) plate, $\mathcal{C}_{\|}(0,0)$, however tends to a constant predominantly determined by the forcing spectral density as the inter-plate separation is increased. To eliminate such spurious effects, we subtract this limiting value that represents the self-interaction of the mobile plate in the bulk by defining the excess correlator as $\Delta \mathcal{C}_{\|}(0,0)=\mathcal{C}_{\|}(0,0)-\lim _{h_{\|} \rightarrow \infty} \mathcal{C}_{\|}(0,0)$. In all cases, our numerical results remain independent of the choice of the frequency cutoff, when the latter is large enough, but it is still kept within the regime consistent with the continuum hydrodynamic description (Appendix B).

As the log-log plots in Fig. 4 show, the stress correlators fall off rapidly as power-laws of the rescaled interplate separation, $h_{\|}$, in all cases (panels a to c), when $h_{\|}$is sufficiently large. In fact, one can discern distinct power-law regimes for both small and large values of $h_{\|}$ as $\gamma_{\|}$is varied. As noted before, the key parameter here is the ratio $h_{\|} / \gamma_{\|}$. We find the power-law behaviors as

$$
\begin{aligned}
& \mathcal{C}_{\|}\left(h_{\|}, h_{\|}\right) \sim \begin{cases}h_{\|}^{-2} & h_{\|} / \gamma_{\|} \gg 1, \\
h_{\|}^{-1} & h_{\|} / \gamma_{\|} \ll 1,\end{cases} \\
& \Delta \mathcal{C}_{\|}(0,0) \sim \begin{cases}h_{\|}^{-4} & h_{\|} / \gamma_{\|} \gg 1, \\
h_{\|}^{-1} & h_{\|} / \gamma_{\|} \ll 1,\end{cases} \\
& \mathcal{C}_{\|}\left(0, h_{\|}\right) \sim \begin{cases}h_{\|}^{-3} & h_{\|} / \gamma_{\|} \gg 1, \\
h_{\|}^{-1} & h_{\|} / \gamma_{\|} \ll 1 .\end{cases}
\end{aligned}
$$

Because of the cumbersome form of the integrand in Eq. (39) (involving factors with implicit and complex-valued dependence on $\left.\omega_{\|}\right)$, we were not able to derive the scaling forms analytically. They have rather been confirmed numerically, with the reported universal exponents being accurate within a margin of error $<5 \%$. These power-laws reflect the more fundamental scale-invariant forms admitted in general by each of the above transverse 

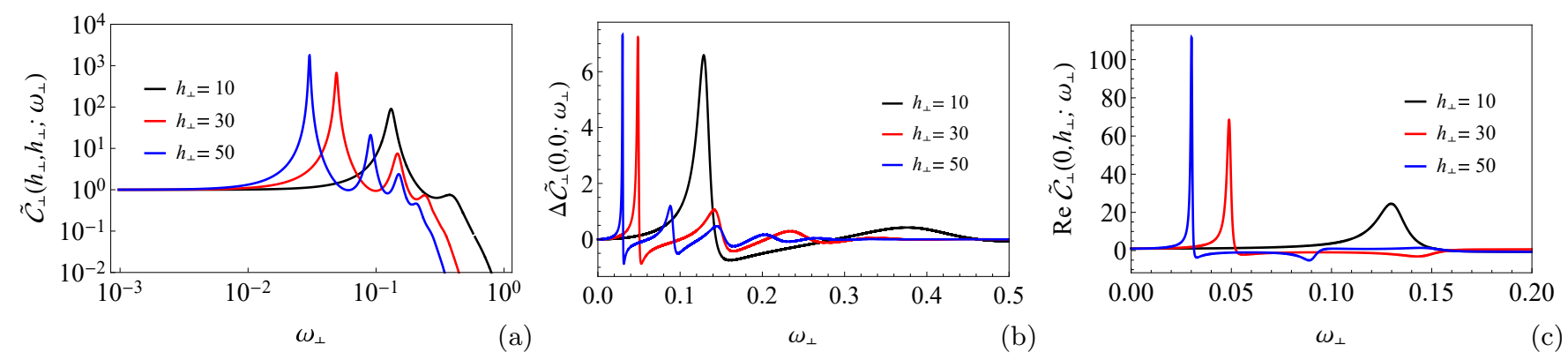

FIG. 6. Log-log plot of the longitudinal stress self-correlator $\widetilde{\mathcal{C}_{\perp}}\left(h_{\perp}, h_{\perp} ; \omega_{\perp}\right)$ in panel (a), and linear plots of the excess longitudinal stress (self- and cross-) correlators $\Delta \widetilde{\mathcal{C}}_{\perp}\left(0,0 ; \omega_{\perp}\right)$ and $\operatorname{Re} \widetilde{\mathcal{C}}_{\perp}\left(0, h_{\perp} ; \omega_{\perp}\right)$ in panels (b) and (c), respectively, as functions of the dimensionless frequency, $\omega_{\perp}$, for fixed $\gamma_{\perp}=2$ and $h_{\perp}=10,30$ and 50 as indicated on the graphs.

stress correlators in terms of their two main parameters as $\mathcal{C}_{\|}=h_{\|}^{-2} \mathcal{F}\left(\gamma_{\|} / h_{\|}\right)$, where $\mathcal{C}_{\|}$stands for either of the quantities $\mathcal{C}_{\|}\left(h_{\|}, h_{\|}\right), \Delta \mathcal{C}_{\|}(0,0)$ or $\mathcal{C}_{\|}\left(0, h_{\|}\right)$, and $\mathcal{F}(\cdot)$ is the corresponding universal function. For these three cases, the universal functions can be calculated numerically for a wide range (several decades) of their arguments with the results, shown in the log-log plot of Fig. 5. clearly demonstrating the crossover between two distinct powerlaw regimes: These regimes appear as straight lines for small and large $h_{\|} / \gamma_{\|}$for each one of the three plotted curves. The numerical values of the slopes match the corresponding exponents given in Eqs. 45-47) plus two.

\section{LONGITUDINAL STRESS CORRELATORS}

The longitudinal stress correlators show distinct features as compared with their transverse counterparts. As seen from the frequency-domain plots in panels a to $\mathrm{c}$ in Fig. 6 the longitudinal self- and cross-correlators exhibit well-developed peaks, representing acoustic resonances due to the compressional modes excited by the external surface forcing in the fluid film. These modes are associated with the poles of the corresponding response functions in the complex frequency plane (see Appendix A). The peaks observed along the real-frequency axis, $\omega_{\perp}$, in Fig. 6 are indeed produced by the first few compression poles (for instance, the four peaks seen for $h_{\perp}=50$, blue curve, in $\widetilde{\mathcal{C}}_{\perp}\left(h_{\perp}, h_{\perp} ; \omega_{\perp}\right)$, panel a, coincide with the loci of the first four poles with $0 \leq n \leq 3$ ). The number of peaks and their heights grow and their loci shift to smaller frequencies as the inter-plate separation, $h_{\perp}$, is increased (and/or as $\gamma_{\perp}$ is increased). At a given inter-plate separation, the longitudinal stress correlators take their largest values at the first peak, which gives the dominant contribution to the frequency integrals. This signifies the prevalence of low-frequency acoustic resonances at larger inter-plate separations and the major role of the corresponding acoustic modes propagating across the film in intensifying fluctuations and correlations of the longitudinal stresses exerted on the confining plates (the higherorder acoustic modes are more strongly attenuated as

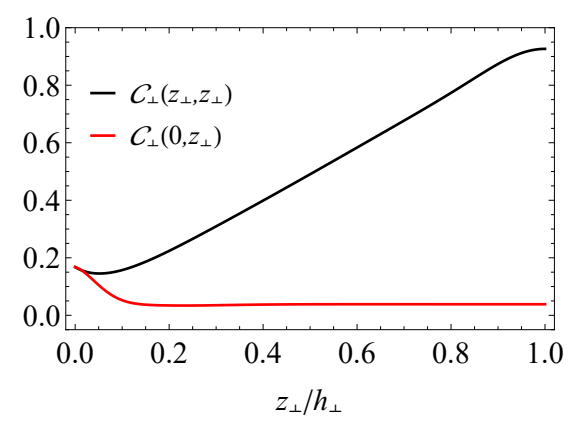

FIG. 7. Profiles of the equal-time longitudinal stress correlators $\mathcal{C}_{\perp}\left(z_{\perp}, z_{\perp}\right)$ and $\mathcal{C}_{\perp}\left(0, z_{\perp}\right)$ across the fluid film $\left(0 \leq z_{\perp} \leq\right.$ $\left.h_{\perp}\right)$ for fixed $h_{\perp}=50$ and $\gamma_{\perp}=2$.

higher-order peak heights are suppressed by one or even few orders of magnitude as seen in panel a).

Although, such acoustic resonances strengthen the longitudinal stress correlator on the upper (fixed) plate, $\widetilde{\mathcal{C}_{\perp}}\left(h_{\perp}, h_{\perp} ; \omega_{\perp}\right)$, which thus remains consistently above its zero-frequency value of one for a wide range of frequencies, sound absorption becomes gradually dominant (as the imaginary parts of the compression poles become large; Appendix A, causing the stress correlator to fall off to zero at sufficiently high frequencies (for $h_{\perp}=50$ in panel a, this occurs on approach, but well before, the chosen upper frequency cutoff of $\omega_{\perp}^{\infty}=1$; see Appendix B. In the case of $\Delta \widetilde{\mathcal{C}_{\perp}}\left(0,0 ; \omega_{\perp}\right)$ and $\widetilde{\mathcal{C}}_{\perp}\left(0, h_{\perp} ; \omega_{\perp}\right)$ in panels $\mathrm{b}$ and $\mathrm{c}$ in Fig. 6, the correlators can take both positive and negative values. The negative values of these quantities represent out-of-phase (or anti-) correlations occurring in certain intervals along the real-frequency axis.

\section{A. Equal-time longitudinal stress correlators}

The equal-time longitudinal stress correlators defined through Eq. [40], and denoted more compactly as 

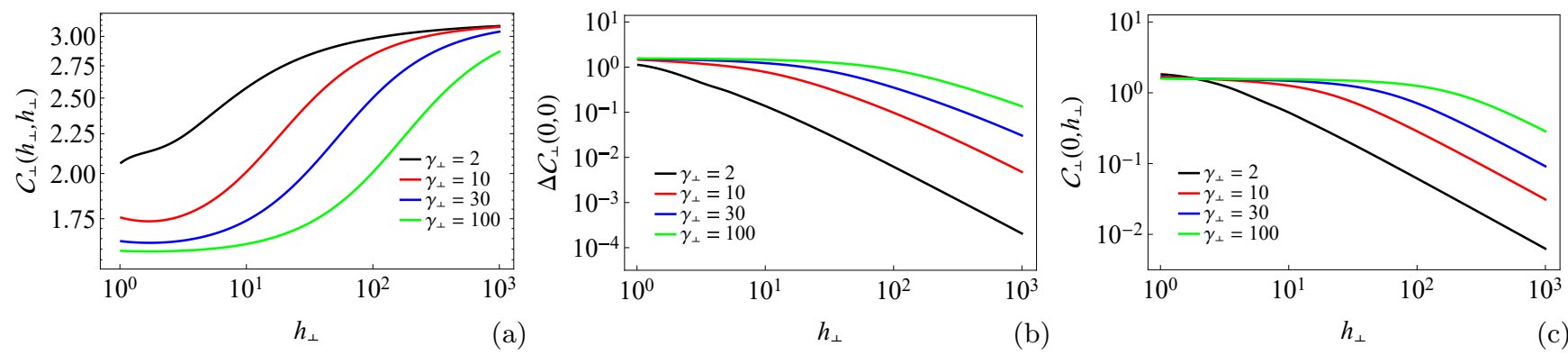

FIG. 8. Log-log plots of equal-time longitudinal stress (self- and cross-) correlators $\mathcal{C}_{\perp}\left(h_{\perp}, h_{\perp}\right), \Delta \mathcal{C}_{\perp}(0,0)$ and $\mathcal{C}_{\perp}\left(0, h_{\perp}\right)$, in panels (a), (b) and (c), respectively, as functions of the dimensionless inter-plate separation, $h_{\perp}$, for $\gamma_{\perp}=2,10,30$ and 100 .

$$
\begin{gathered}
\mathcal{C}_{\|}\left(z_{\perp}, z_{\perp}^{\prime}\right) \equiv \mathcal{C}_{\|}\left(z_{\perp}, z_{\perp}^{\prime} ; \Delta \tau_{\perp}=0\right), \text { can be evaluated from } \\
\qquad \mathcal{C}_{\perp}\left(z_{\perp}, z_{\perp}^{\prime}\right)=\int \frac{\mathrm{d} \omega_{\perp}}{2 \pi} \widetilde{\chi}_{\perp}\left(z_{\perp} ; \omega_{\perp}\right) \widetilde{\chi}_{\perp}^{*}\left(z_{\perp}^{\prime} ; \omega_{\perp}\right)
\end{gathered}
$$

Unlike their transverse counterparts, these correlators do not in general admit scale-invariant forms. In Fig. 7 , we show $\mathcal{C}_{\perp}\left(z_{\perp}, z_{\perp}\right)$ and $\mathcal{C}_{\perp}\left(0, z_{\perp}\right)$ as functions of $z_{\perp} / h_{\perp}$ for fixed $h_{\perp}=50$ and $\gamma_{\perp}=2$. It is interesting to note that while the different-point $\left(z_{\perp} \neq z_{\perp}^{\prime}\right)$ correlator levels off rapidly to a limiting value smaller than the reference value of $\mathcal{C}_{\perp}(0,0)$, the same-point correlator of longitudinal stresses increases almost linearly as one moves away from the lower (mobile) plate toward the upper (fixed) plate, where it takes its largest value. This indicates stronger hydrodynamic stress fluctuations closer to the fixed plate and pronounced, non-decaying, r.m.s. values for the longitudinal stresses acting on it even at relatively large separations.

This non-decaying behavior is more clearly seen from the plot in panel a in Fig. 8. Here, the equal-time longitudinal correlator, $\mathcal{C}_{\perp}\left(h_{\perp}, h_{\perp}\right)$, is shown to increase to a saturated maximum level, depending on the dimensionless mass parameter $\gamma_{\perp}$. The reason for this behavior is that as $h_{\perp}$ is gradually increased (starting from its minimum base value of $h_{\perp}=1$ ), the characteristic frequency corresponding to the first compressional mode decreases and falls below the cutoff frequency of $\omega_{\perp}^{\infty}=1$, making it realizable and relevant in the hydrodynamic domain, manifesting itself also as a gradual increase in $\mathcal{C}_{\perp}\left(h_{\perp}, h_{\perp}\right)$. As $h_{\perp}$ is further increased, the first characteristic frequency (whose locus over the real-frequency axis scales as $h_{\perp}^{-1}$, as is characteristic to acoustic modes) decreases further toward the low-frequency regions, where sound attenuation is subdominant. As such, the corresponding acoustic resonance leads to a strongly propagating acoustic mode across the fluid film, creating a pronounced first peak in the stress-correlator plots in the frequency domain (panel a in Fig. 6), bringing $\mathcal{C}_{\perp}\left(h_{\perp}, h_{\perp}\right)$ up to a saturation level (panel a in Fig. 8, see also panel b in Fig. 10 in Appendix B). The crossover to the saturation regime in $\mathcal{C}_{\perp}\left(h_{\perp}, h_{\perp}\right)$ occurs roughly at $h_{\perp} \sim \gamma_{\perp}$.

In the case of the longitudinal stress correlators $\Delta \mathcal{C}_{\perp}(0,0)$ and $\mathcal{C}_{\perp}\left(0, h_{\perp}\right)$, shown in panels b and $\mathrm{c}$, and in analogy with our discussion of the power-law behaviors in the case of transverse correlators, we find power-law behaviors in the regime of large inter-plate separations, $h_{\perp} / \gamma_{\perp} \gg 1$, with universal exponents as

$$
\begin{aligned}
& \Delta \mathcal{C}_{\perp}(0,0) \sim h_{\perp}^{-3 / 2}, \\
& \mathcal{C}_{\perp}\left(0, h_{\perp}\right) \sim h_{\perp}^{-1} .
\end{aligned}
$$

The excess stress correlator on the lower (mobile) plate is defined here as $\Delta \mathcal{C}_{\perp}(0,0)=\mathcal{C}_{\perp}(0,0)-\lim _{h_{\perp} \rightarrow \infty} \mathcal{C}_{\perp}(0,0)$.

In all cases discussed above, the main contribution to the longitudinal correlators comes from the fluctuations and correlation produced in the film through the pressure (second) term in Eq. (9) rather than the viscous stress (first) terms.

\section{DISCUSSION AND CONCLUSION}

We have studied hydrodynamic correlations and fluctuation-induced interactions mediated between the no-slip bounding surfaces of a planar film of a compressible and viscous fluid, driven externally at one of its boundaries (lower plate) by a stochastic surface forcing of arbitrary spectral density, while the other (upper) plate is kept fixed. We develop general analytical results within the linear hydrodynamic scheme and numerically analyze the outcomes for the special case of a Gaussian white-noise forcing. The stochastic surface forcing leads to fluctuating transverse (shear) and longitudinal (compressional) hydrodynamic stresses within the film and on the bounding surfaces. To bring out the hydrodynamic fluctuation-induced effects more clearly, we conveniently assume that the external forcing has a zero mean; hence, the resulting hydrodynamic stresses also vanish on average, and their two-point correlators embody the hydrodynamic correlation effects.

We show that the same-plate (self-) and cross-plate (cross-) correlators of the transverse stress exhibit two distinct regimes of power-law behaviors at small and large inter-plate separations $h$, with different, yet universal, scaling exponents. In the case of longitudinal stress correlators, the power-law dependencies are obtained only for the large-separation behavior of the (excess) self-correlator on the mobile plate, $\Delta \mathcal{C}_{\perp}(0,0)$, and 
the cross-correlator, $\mathcal{C}_{\perp}(0, h)$, with power-law decays being expressively weaker than those of the transverse correlators. The spectral analysis of the longitudinal stress correlators reveals distinct underlying differences with the transverse ones due to the prevalence of propagating, underdamped, acoustic modes in the confined geometry [10]. The longitudinal stress self-correlator at the fixed plate, $\mathcal{C}_{\perp}(h, h)$, thus displays a thoroughly different behavior: It increases with $h$ and saturates at a finite value, representing a constant, longitudinal, r.m.s. stress $\sigma_{\perp}^{\mathrm{rms}}=\sqrt{\mathcal{C}_{\perp}(h, h)}$ at sufficiently large $h$. This feature indicates the existence of non-decaying, longitudinal, hydrodynamic fluctuation forces, acting on the fixed plate. This can be contrasted with, e.g., the transverse stresses on the fixed plate, whose r.m.s. decays with the interplate separation as $\sigma_{\|}^{\mathrm{rms}} \sim h^{-1}$ for large $h$.

The non-decaying behavior of $\mathcal{C}_{\perp}(h, h)$ emanates directly from the excitation of the acoustic modes (acoustic resonances) and sets in at the appearance of the first peak in the corresponding spectral representation (Section $\mathrm{V}$. Indeed, one can directly verify that the dominant contribution to $\mathcal{C}_{\perp}(h, h)$ comes from the compressional term in the longitudinal stress (second term in Eq. (9)). Such long-ranged, sound-mediated, hydrodynamic correlations have also been found in the context of the correlations between Brownian particles (colloids) in strongly confined quasi-one/two-dimensional geometries (see Ref. [10] and references therein; see also Ref. [11] for recent experiments on non-decaying colloid-colloid correlations based on the displacement of the intervening fluid column between the colloids in narrow channels). It should be further noted that our analysis is focused on the stationary-state behavior of the system, implying that the film thickness is traversed by recurring propagations of underdamped sound waves of varying (random) amplitude, continually excited by the longitudinal component of the external forcing applied to the mobile plate. This process generates and maintains a finite and stationary (non-decaying) compressional r.m.s. stress on the fixed plate.

The power-law relations obtained here for the (generally non-thermal) surface-driven stress correlators assimilate to the "secondary" hydrodynamic Casimir-like forces, analyzed previously in the context of thermal fluctuations in a fluid film with fixed boundaries 40. Such non-equilibrium fluctuation-induced, or Casimirlike effects have been of considerable interest in other soft-matter contexts in recent years 38 48. It is also important to note that the r.m.s. of the fluctuationinduced forces predicted here exhibits a stronger longranged character than the standard electromagnetic vdW-Casimir forces [6, 8]. In the case of the longitudinal stresses on the fixed plate, our results (panel a of Fig. 8) give a rescaled r.m.s. stress of the order one over the range of rescaled separations $h_{\perp} \sim 10-10^{2}$ for a wide range of values for $\gamma_{\perp}$. Thus, for the parameter values relevant to water (see Appendix B, we predict a longitudinal r.m.s. stress (pressure) of the or- der of the r.m.s. surface forcing, $\sigma_{\perp}^{\mathrm{rms}} / \sqrt{\sigma_{f}} \sim 1$, over the range of inter-plate separations, or film thicknesses, $h \sim 2.6 \times\left(10-10^{2}\right) \mathrm{nm}$. Here, $\sigma_{f}$ stands for the surface forcing variance in the time domain (related to the forcing spectral density as $\sigma_{f}=2 \mathcal{G}_{z} \omega^{\infty}$; see Eq. (38), Section II C and Appendix B. Being an externally controlled quantity, $\sigma_{f}$ can be adjusted arbitrarily to obtain the experimental resolution required for the verification of our predictions. The force-measurement precision within the dynamic SFA and AFM techniques can be better than $10 \mathrm{nN}$ and $10 \mathrm{pN}$, respectively [1, 2, 13, 16]; hence, in the later case (of thermal-noise AFM), even the effects due to ambient thermal noise have been detectable [2, 13, 14, 27, 29, 31, 33.

Despite its geometric simplicity, our model captures the essential features of typical dynamic SFA/AFM setups [12 35. (see Section II A for further details). While these techniques have widely been used in the study of the rheological properties of fluid films, substantial focus has been on their utility in high-precision determination of shear/compressional forces produced on the bounding surfaces confining a fluid film, where one of the surfaces is forced in linear or oscillatory motion. In dynamic SFA, the bounding surfaces are usually taken as two apposed, weakly curved, crossed cylinders (sometimes with large flattened contact areas exposed to the intervening fluid 1, 2, 12, 20, 22, 34]), or a sphere and a plane, with the radii of curvature in either case (of the order of a few $\mathrm{mm}$ or $\mathrm{cm}$ ) being much larger than the film thickness (in the range of sub-nm to $\mu \mathrm{m}$, or larger) [12, 15, 16, 2026]. In dynamic AFM, wide flat microlevers [30], or relatively large, cantilever-mounted spheres (of radius up to tens of $\mu \mathrm{m})$ [2, 13, 16, 27, 28, 32, 33] are used in forced oscillations or in spontaneous (thermal) stochastic motions next to a planar substrate, probing the local surface properties, the hydrodynamic/viscoelastic properties of the surrounding fluid, and also the hydrodynamic interactions mediated between the probe and the substrate. The typical film geometries and modes of surface motions employed in the aforementioned setups therefore appear well-suited for testing our theoretical predictions. The main assumptions made within our analytical approach (e.g., using small-amplitude oscillations and linearized hydrodynamic treatment) are also directly relevant to the mentioned experimental setups and also agree with previous modeling approaches [12 16, 24, 33]. To the best our knowledge, however, the surface-force experiments have so far been focused merely on the net force mediated between the fluid film boundaries as opposed to force fluctuations and correlations. The predicted behaviors for these latter quantities can be examined by scrutinizing the readily available r.m.s. of the experimentally detected forces as a function of the film thickness.

Our work thus lays out a self-consistent and systematic hydrodynamic-fluctuations approach, incorporating the often-ignored finite compressibility of the fluid film, which is important in the understanding of the sound-mediated effects. It also places the study of hy- 
drodynamic surface forces induced by externally driven thin films within the newly emerging context of nonequilibrium Casimir-type phenomena.

By connecting the spectral density of hydrodynamic stresses acting on the surface boundaries and the spectral density of fluid density fluctuations in the film (see Eq. (29)), our analysis also suggests an alternative method to probe the density fluctuations through the measurements of surface forces in confined fluid films, where standard light scattering methods may be less suitable.

Finally, we note that our numerical results are given here only for the case of white-noise forcing. Other examples of external forcing (such as colored or $1 / f$ noises) can straightforwardly be analyzed using the more general analytical formulas presented (Section III). Other possible avenues that can be explored within the present context include the surface curvature effects, the fluid thermal conductivity [53] and possibly also the role of nonlinear effects such as viscous dissipation [23.

\section{ACKNOWLEDGEMENTS}

M.M.-A. and S.M. acknowledge funding from the School of Physics, Institute for Research in Fundamental Sciences (IPM), where the research leading to this publication was performed. A.N. acknowledges partial support from Iran Science Elites Federation (ISEF) and the Associateship Scheme of The Abdus Salam International Centre for Theoretical Physics (Trieste, Italy). R.P. gratefully acknowledges support from the " 1000 Talents Program" of China and from the University of Chinese Academy of Sciences (UCAS).

\section{Appendix A: Hydrodynamic modes}

The transverse (longitudinal) hydrodynamic modes are given by the poles of the transverse (longitudinal) response functions, occurring only in the lower half of the complex frequency $(\varsigma)$ plane, at the roots of the equation,

$$
\cos [\Upsilon(\varsigma)]-\Theta \Upsilon(\varsigma) \sin [\Upsilon(\varsigma)]=0,
$$

where we have used Eqs. (35) and (36) and the definitions

$$
\left\{\begin{array}{lll}
\Theta=\gamma_{\|} / h_{\|}, \Upsilon(\varsigma)=\xi(\varsigma) h_{\|} & : & \text {(shear) } \\
\Theta=\gamma_{\perp} / h_{\perp}, \Upsilon(\varsigma)=\ell(\varsigma) h_{\perp}: & \text { (compression) } .
\end{array}\right.
$$

The parameter $\Upsilon(\varsigma)$ can be expressed in terms of its real and imaginary parts as $\Upsilon(\varsigma)=\Upsilon_{R}(\varsigma)+i \Upsilon_{I}(\varsigma)$.

For shear modes, Eq. A1 admits a series of roots only on the imaginary axis, whose loci can be estimated analytically by looking at two limiting cases. For $\Theta \gg 1$ (large dimensionless mass parameter for the mobile plate, $\gamma_{\|}$, or small dimensionless inter-plate separation, $h_{\|}$) and, on the leading order, Eq. A1 takes the approximate form $\exp [2 i \Upsilon(\varsigma)] \simeq 1$ or, equivalently, $\Upsilon_{R}(\varsigma) \simeq n \pi$ and $\Upsilon_{I}(\varsigma) \simeq 0$, where $n$ is an integer. For $\Theta \ll 1$, we find the

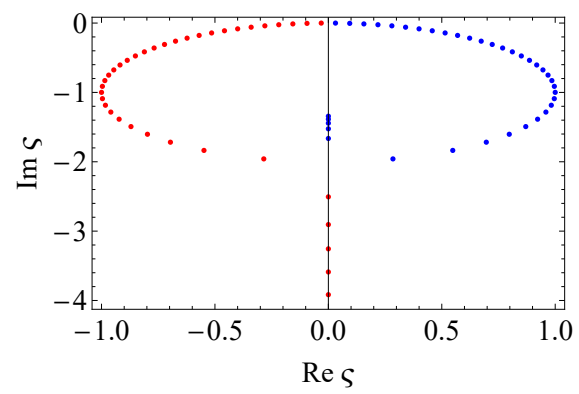

FIG. 9. Poles of the longitudinal stress response functions in the complex frequency $(\varsigma)$ plane for $h_{\perp}=50$ and $\gamma_{\perp}=2$. The poles are shown up to the order $|n| \leq 36$, of which the last four poles fall over the imaginary axis (become purely diffusive). The rest of the poles (shown in two left and right branches in red and blue colors, respectively) represent partially attenuated acoustic modes propagating across the film.

approximate leading-order equations $\Upsilon_{\mathrm{R}}(\varsigma) \simeq(n+1 / 2) \pi$ and $\Upsilon_{I}(\varsigma) \simeq 0$. Using the definitions in Eqs. 32 and A2 , the shear modes are located as

$$
\varsigma_{\|}^{(n)} \simeq-i \frac{\pi^{2}}{h_{\|}^{2}} \times \begin{cases}(n+1 / 2)^{2} & h_{\|} / \gamma_{\|} \gg 1 \\ n^{2}(n \neq 0) & h_{\|} / \gamma_{\|} \ll 1\end{cases}
$$

For compressional modes, the roots of Eq. (A1) can have both finite real and finite imaginary parts. The approximate loci of the roots can be obtained for $\Theta \gg 1$ and $\Theta \ll 1$ using limiting arguments analogous to those discussed above for the shear modes. Using the definitions in Eqs. (33), (34) and (A2), we arrive at $\varsigma^{2}+i \varsigma q_{n}^{2}-q_{n}^{2} \simeq 0$, solving which gives the roots $\varsigma_{\perp}^{(n)}$ as

$$
\varsigma_{\perp}^{(n)} \simeq \pm \frac{\left|q_{n}\right|}{2} \sqrt{4-q_{n}^{2}}-i \frac{q_{n}^{2}}{2},
$$

where we have

$$
q_{n}=\frac{\pi}{h_{\perp}} \times \begin{cases}n+1 / 2 & h_{\perp} / \gamma_{\perp} \gg 1, \\ n(n \neq 0) & h_{\perp} / \gamma_{\perp} \ll 1 .\end{cases}
$$

These poles exhibit a finite real part (representing sound propagation with a finite phase velocity) and a finite imaginary part (representing a finite lifetime due to sound absorption) for $\left|q_{n}\right|<2$, or, equivalently, $\mid n+$ $1 / 2 \mid<2 h_{\perp} / \pi$, when $h_{\perp} / \gamma_{\perp}$ is sufficiently large. These poles exhibit two distinct, mirror symmetric, left and right branches with negative and positive real parts, shown in Fig. 9 by red and blue symbols, respectively. The poles with larger order in $n$ become purely imaginary and the corresponding compressional modes become diffusive. The diffusive poles stemming from the right branch densely accumulate in a short interval just below $-i$, and gradually approach the latter as a limiting value, when $|n| \rightarrow \infty$; while, those stemming from the left branch spread with increasingly large separations over the imaginary axis, tending to $-i \infty$. The real part of the poles never exceeds one in magnitude on either 


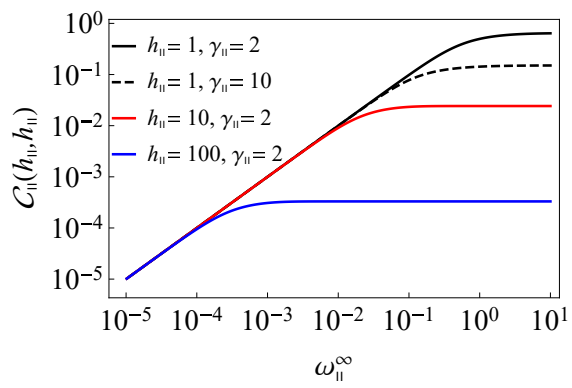

(a)

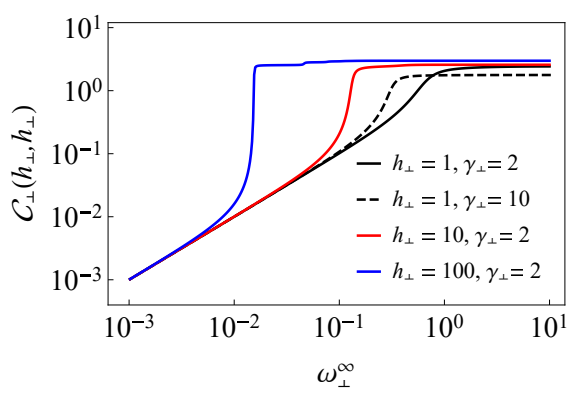

(b)

FIG. 10. Log-log plots of equal-time self-correlators on the fixed plate, $\mathcal{C}_{\|}\left(h_{\|}, h_{\|}\right)$and $\mathcal{C}_{\perp}\left(h_{\perp}, h_{\perp}\right)$, in panels (a) and (b) respectively, as functions of the corresponding dimensionless frequency cutoffs for different values of the dimensionless system parameters as indicated on the graphs.

branch, irrespective of the other parameter values; thus, all of the poles fall within the frequency integration domain $\left|\omega_{\perp}\right| \leq \omega_{\perp}^{\infty}=1$.

\section{Appendix B: Cutoff and other parameter values}

Although the frequency integrals appearing in the analytical results are originally defined over the whole real axis, it is clear that the hydrodynamic description breaks down for very large frequencies, or at very small lengthscales below a microscopic cutoff $a$. In our numerical analysis, we impose an upper frequency cutoff $\omega^{\infty}$ and take the integrals over the range $|\omega| \leq \omega^{\infty}$; for consistency, we also limit the range of film thicknesses to $h \geq a$. Nevertheless, it is important to note that the frequency integrals in our analysis of the stress correlators, Eqs. (44) and 48, are found to converge sufficiently rapidly, giving finite results as the cutoff is taken to infinity. The introduction of a cutoff is important as it enables us to ensure that our results are primarily determined by the frequencies that fall within the hydrodynamic regime.

For the most part, we set the microscopic length-scale cutoff $a=\nu_{\|, \perp} / c_{0}$ with the corresponding frequency cutoff $\omega^{\infty}=c_{0}^{2} / \nu_{\|, \perp}$ for the shear/compression modes. In dimensionless units, we have $a_{\|, \perp}=1$ and $\omega_{\|, \perp}^{\infty}=1$ (Section III C). For the parameter values relevant to water, e.g., $\eta \simeq 8.9 \times 10^{-4} \mathrm{~Pa} \cdot \mathrm{s}, \rho_{0} \simeq 997 \mathrm{Kg} \cdot \mathrm{m}^{-3}, c_{0} \simeq 1496.7 \mathrm{~m} \cdot \mathrm{s}^{-1}$ at $25^{\circ} \mathrm{C} 57$ and assuming $\zeta \simeq 3 \eta$ [58, the lengthscale and frequency cutoffs for the shear (compression) modes are found approximately $a \simeq 0.6 \mathrm{~nm}(2.6 \mathrm{~nm})$ and $\omega^{\infty} \simeq 2.5 \times 10^{12} \mathrm{~s}^{-1}\left(0.6 \times 10^{12} \mathrm{~s}^{-1}\right)$, respectively. These values agree with recent studies indicating validity of the continuum hydrodynamic description in water down to scales of around one nanometer [19]. In other words, in the present context, taking the dimensionless frequency cutoffs larger than $\omega_{\|, \perp}^{\infty} \sim 1$ may not be justified.

We show the exemplary cases of equal-time selfcorrelators on the fixed plate, $\mathcal{C}_{\|}\left(h_{\|}, h_{\|}\right)$and $\mathcal{C}_{\perp}\left(h_{\perp}, h_{\perp}\right)$, as functions of the corresponding dimensionless frequency cutoffs in Fig. 10 for selected values of the dimensionless system parameters as indicated on the graphs. As seen, the plotted quantities saturate to a limiting cutoffindependent plateau when the corresponding cutoff is large enough. For the slowest rate of convergence to the plateau levels, which is obtained for the lowest admissible value of the inter-plate separation $h_{\|}=h_{\perp}=1($ or $h=a)$, we find that the saturations in $\mathcal{C}_{\|}\left(h_{\|}, h_{\|}\right)$and $\mathcal{C}_{\perp}\left(h_{\perp}, h_{\perp}\right)$ occur roughly around $\omega_{\|}^{\infty}=\omega_{\perp}^{\infty}=1$, when $\gamma_{\|}=\gamma_{\perp}=2$. The results for larger values of $h_{\|}$and $h_{\perp}$ and/or larger values of $\gamma_{\|}$and $\gamma_{\perp}$ show faster convergence trends to their cutoff-independent values. The saturations occur roughly at $\omega_{\|}^{\infty} \sim h_{\|}^{-2}\left(\omega_{\perp}^{\infty} \sim h_{\perp}^{-1}\right)$ for the transverse (longitudinal) correlators. These observations confirm that our numerical choices for the length/frequency cutoffs are consistent with those expected experimentally [19] and, yet, they are large enough to give the desired saturation (plateau) values for the numerical outcomes.

It is also to be noted that for longitudinal correlators, the saturation is rather abrupt, occurring when the cutoff $\omega_{\perp}^{\infty}$ exceeds the locus of the first peak along the realfrequency axis (panel a in Fig. 6). In other words, the longitudinal frequency integrals quickly saturate to their cutoff-independent values as soon as the integration domain, $\left|\omega_{\perp}\right| \leq \omega_{\perp}^{\infty}$, is wide enough to include the first peak (first excited acoustic mode), as this peak gives the major dominant contribution to the frequency integrals.
[1] J. N. Israelachvili, Intermolecular and Surface Forces, 3rd ed. (Academic Press, Amsterdam, 2011).

[2] H.-J. Butt and M. Kappl, Surface and Interfacial Forces (Wiley-VCH, New York, 2010).

[3] J. Happel and H. Brenner, Low Reynolds number hydrodynamics: with special applications to particulate media, (Springer Netherlands \& Martinus Nijhoff Publish- ers, The Hague, 1983).

[4] J. K. G. Dhont, An Introduction to Dynamics of Colloids (Elsevier Science, Amsterdam, 1996).

[5] D. S. Dean, J. Dobnikar, A. Naji, and R. Podgornik, Electrostatics of Soft and Disordered Matter (Pan Stanford Publishing, Singapore, 2014).

[6] L. M. Woods, D. A. R. Dalvit, A. Tkatchenko, P. 
Rodriguez-Lopez, A. W. Rodriguez, and R. Podgornik, Materials perspective on Casimir and van der Waals interactions, Rev. Mod. Phys. 88, 045003 (2016).

[7] C. Stanley and D. C. Rau, Evidence for water structuring forces between surfaces, Curr. Opin. Colloid Interface Sci. 16, 551 (2011).

[8] M. Kardar and R. Golestanian, The "friction" of vacuum and other fluctuation-induced forces, Rev. Mod. Phys. 71, 1233 (1999).

[9] V. Mkrtchian, V. A. Parsegian, R. Podgornik, and W. M. Saslow, Universal thermal radiation drag on neutral objects, Phys. Rev. Lett. 91, 220801 (2003).

[10] D. Frydel and H. Diamant, Long-range dynamic correlations in confined suspensions, Phys. Rev. Lett. 104, $248302(2010)$.

[11] K. Misiunas, S. Pagliara, E. Lauga, J. R. Lister, and U. F. Keyser, Nondecaying hydrodynamic interactions along narrow channels, Phys. Rev. Lett. 115, 038301 (2015).

[12] J. Israelachvili, Y. Min, M. Akbulut, A. Alig, G. Carver, W. Greene, K. Kristiansen, E. Meyer, N. Pesika, K. Rosenberg, and H. Zeng, Recent advances in the surface forces apparatus (SFA) technique, Rep. Prog. Phys. 73, 036601 (2010).

[13] H.-J. Butt, B. Capella, and M. Kappl, Force measurements with the atomic force microscope: Technique, interpretation and applications, Surf. Sci. Rep. 59, 1 (2005).

[14] D. B. Haviland, Quantitative force microscopy from a dynamic point of view, Curr. Opin. Colloid Interface Sci. 27, 74 (2017).

[15] Y. Wang, G. A. Pilkington, C. Dhong, and J. Frechette, Elastic deformation during dynamic force measurements in viscous fluids, Curr. Opin. Colloid Interface Sci. 27, 43 (2017).

[16] J. Huang, B. Yan, A. Faghihnejad, H. Xu, and H. Zeng, Understanding nanorheology and surface forces of confined thin films, Korea-Aust. Rheol. J. 26, 3 (2014).

[17] J. S. Ellis and M. Thompson, Slip and coupling phenomena at the liquid-solid interface, Phys. Chem. Chem. Phys. 6, 4928 (2004).

[18] C. Neto, D. R. Evans, E. Bonaccurso, H.-J. Butt, and V. S. J. Craig, Boundary slip in Newtonian liquids: a review of experimental studies, Rep. Prog. Phys. 68, 2859 (2005).

[19] L. Bocquet and E. Charlaix, Nanofluidics, from bulk to interfaces, Chem. Soc. Rev. 39, 1073 (2010).

[20] J. Peachey, J. Van Alsten, and S. Granick, Design of an apparatus to measure the shear response of ultrathin liquid films, Rev. Sci. Instrum. 62, 463 (1991).

[21] E. Kumacheva and J. Klein, Simple liquids confined to molecularly thin layers. II. Shear and frictional behavior of solidified films, J. Chem. Phys. 108, 7010 (1998).

[22] L. Bureau, Nonlinear rheology of a nanoconfined simple fluid, Phys. Rev. Lett. 104, 218302 (2010).

[23] J. Klein, Frictional dissipation in stick-slip sliding, Phys. Rev. Lett. 98, 056101 (2007).

[24] C. Cottin-Bizonne, A. Steinberger, B. Cross, O. Raccurt, and E. Charlaix, Nanohydrodynamics: The intrinsic flow boundary condition on smooth surfaces, Langmuir 24, 1165 (2008).

[25] A. Steinberger, C. Cottin-Bizonne, P. Kleimann, and E. Charlaix, Nanoscale flow on a bubble mattress: Effect of surface elasticity, Phys. Rev. Lett. 100, 134501 (2008).

[26] S. Leroy, A. Steinberger, C. Cottin-Bizonne, F. Restagno,
L. Léger, and E. Charlaix, Hydrodynamic interaction between a spherical particle and an elastic surface: A gentle probe for soft thin films, Phys. Rev. Lett. 108, 264501 (2012).

[27] F. Benmouna and D. Johannsmann, Hydrodynamic interaction of AFM cantilevers with solid walls: An investigation based on AFM noise analysis, Eur. Phys. J. E 9, 435 (2002).

[28] J. Alcaraz, L. Buscemi, M. Puig-de-Morales, J. Colchero, A. Baro, and D. Navajas, Correction of microrheological measurements of soft samples with atomic force microscopy for the hydrodynamic drag on the cantilever, Langmuir 18, 716 (2002).

[29] R. J. Clarke, S. M. Cox, P. M. Williams, and O. E. Jensen, The drag on a microcantilever oscillating near a wall, J. Fluid Mech. 545, 397 (2005).

[30] A. Siria, A. Drezet, F. Marchi, F. Comin, S. Huant, and J. Chevrier, Viscous cavity damping of a microlever in a simple fluid, Phys. Rev. Lett. 102, 254503 (2009).

[31] C. A. Van Eysden and J. E. Sader, Frequency response of cantilever beams immersed in compressible fluids with applications to the atomic force microscope, J. Appl. Phys. 106, 094904 (2009).

[32] A. Maali, R. Boisgard, H. Chraibi, Z. Zhang, H. Kellay, and A. Würger, Viscoelastic drag forces and crossover from no-slip to slip boundary conditions for flow near airwater interfaces, Phys. Rev. Letts. 118, 084501 (2017).

[33] Y. Wang, B. Zeng, H. T. Alem, Z. Zhang, E. Charlaix, and A. Maali, Visco-capillary response of gas bubbles probed by thermal noise atomic force measurement, Langmuir 30, 1371 (2018).

[34] S. Berg, M. Ruths, and D. Johannsmann, Quartz crystal resonators with atomically smooth surfaces for use in contact mechanics, Rev. Sci. Instrum. 74, 3845 (2003).

[35] D. G. Grier and S. H. Behrens, in Electrostatic Effects in Soft Matter and Biophysics, Eds. C. Holm, P. Kékicheff, and R. Podgornik, NATO Science Series, Vol. 46 (Springer Netherlands, Dordrecht, 2001).

[36] A. Erbaş, R. Podgornik, and R. R. Netz, Viscous compressible hydrodynamics at planes, spheres and cylinders with finite surface slip, Eur. Phys. J. E 32, 147 (2010).

[37] L. D. Landau and E. M. Lifshitz, Fluid Mechanics, 2nd ed. (Butterworth-Heinemann, 1987); Statistical Physics, Part 2 (Butterworth-Heinemann, 1980).

[38] R. B. Jones, Hydrodynamic fluctuation forces, Physica A 105, 395 (1981).

[39] D. Y. C. Chan, L. R. White, On the existence of hydrodynamic fluctuation forces, Physica A 122, 505 (1983).

[40] C. Monahan, A. Naji, R. Horgan, B.-S. Lu, and R. Podgornik, Hydrodynamic fluctuation-induced forces in confined fluids, Soft Matter 12, 441 (2016).

[41] D. Bartolo, A. Ajdari, J.-B. Fournier, and R. Golestanian, Fluctuations of fluctuation-induced Casimir-like forces, Phys. Rev. Lett. 89, 230601 (2002).

[42] D. S. Dean, V. A. Parsegian, and R. Podgornik, Fluctuation of thermal van der Waals forces due to dipole fluctuations, Phys. Rev. A 87, 032111 (2013).

[43] M. Antezza, L. P. Pitaevskii, S. Stringari, and V. B. Svetovoy, Casimir-Lifshitz force out of thermal equilibrium, Phys. Rev. A 77, 022901 (2008).

[44] M. Krüger, T. Emig, and M. Kardar, Nonequilibrium electromagnetic fluctuations: Heat transfer and interactions, Phys. Rev. Lett. 106, 201404 (2011).

[45] T. R. Kirkpatrick, J. M. Ortiz de Zárate, and J. V. Sen- 
gers, Giant Casimir effect in fluids in nonequilibrium steady states, Phys. Rev. Lett. 110, 235902 (2013).

[46] T. R. Kirkpatrick, J.M. Ortiz de Zárate, and J. V. Sengers, Fluctuation-induced pressures in fluids in thermal nonequilibrium steady states, Phys. Rev. E 89, 022145 (2014).

[47] D. S. Dean and A. Gopinathan, Out-of-equilibrium behavior of Casimir-type fluctuation-induced forces for free classical fields, Phys. Rev. E 81, 041126 (2010).

[48] A. Aminov, Y. Kafri, and M. Kardar, Fluctuationinduced forces in nonequilibrium diffusive dynamics, Phys. Rev. Lett. 114, 230602 (2015).

[49] G. T. Silva and H. Bruus, Forces acting on a small particle in an acoustical field in a thermoviscous fluid, Phys. Rev. E 92, 043010 (2015).

[50] T. G. Leighton, The Acoustic Bubble (Academic Press, London, 1994).

[51] To avoid any possible inconsistencies, we assume that the supremum norms of the surface velocity $|\mathbf{u}|$, external surface forcing $|\mathbf{f}|$ and film thickness variations $|\delta h|$ are all sufficiently small and of the same order. The film thickness variations $(|\delta h| \ll h)$ can be shown to contribute only to the subleading terms in our calculations.
[52] Strictly speaking, ignoring local temperature variations and heat transfer processes is equivalent to setting the thermal conductivity coefficient, the isobaric thermal expansion coefficient and specific heat at constant volume equal to zero 37, 55, 56. It turns out, however, that only setting the isobaric thermal expansion coefficient equal to zero is enough to ensure that the local temperature does not vary up to the leading order in fluctuations.

[53] C. Monahan, M. Mohammadi-Arzanagh, A. Naji, B.-S. Lu, and R. Podgornik; to be submitted (2017).

[54] We note the typographic error in Ref. 40, referring to $c_{0}$ as adiabatic speed of sound.

[55] J. P. Boon and S. Yip, Molecular Hydrodynamics (Dover Publications, New York, 1991).

[56] B. J. Berne and R. Pecora, Dynamic Light Scattering: with Applications to Chemistry, Biology, and Physics (Dover Publications, New York, 2000).

[57] W. M. Haynes, D. R. Lide, and T. J. Bruno, CRC Handbook of Chemistry and Physics, 97th Ed. (CRC Press, New York, 2017).

[58] A. S. Dukhin and P. J. Goetz, Bulk viscosity and compressibility measurement using acoustic spectroscopy, J. Chem. Phys. 130, 124519 (2009). 